\title{
O Acolhimento oferecido pelos Serviços de Saúde às pessoas com Necessidades Especiais
}

\author{
Rubenice Gonçalves Gotado ${ }^{1}$; Maria Antonieta Pereira Tigre Almeida ${ }^{2}$
}

\begin{abstract}
Resumo: Objetivou-se analisar as diversas formas de acolhimento prestadas aos usuários com necessidades especiais que utilizam os serviços de saúde da cidade de Vitória da Conquista. Os dados foram obtidos através da aplicação de um questionário objetivo aplicado aos familiares de pessoas com necessidades especiais. A pesquisa é de caráter exploratório, de abordagem quantitativa. A análise foi realizada através do software Microsoft Excel 2013, obtendo os seguintes resultados: grau de parentesco Pai/Mãe (85\%), deficiência mental (45\%), período de espera não é nada razoável (75\%), os familiares raramente se sentiam respeitados (60\%), o acolhimento da equipe é mais ou menos (65\%) e a qualidade do serviço é mais ou menos (95\%). Observou-se que o tempo de atendimento, período de espera, dificuldade para obter informações, serviços inapropriados, escuta e acolhimento inadequados são os principais fatores responsáveis pelas dificuldades do acolhimento oferecido pelos serviços de saúde aos usuários com deficiência.
\end{abstract}

Palavras-chave: Acolhimento; Acesso aos serviços de saúde; Satisfação dos usuários.

\section{The Reception offered by the Health Services to People with Special Needs}

\begin{abstract}
The objective was to analyze the different forms of reception provided to users with special needs who use the health services of the city of Vitória da Conquista. The data were obtained through the application of an objective questionnaire applied to the families of people with special needs. The research is exploratory, with a quantitative approach. The analysis was performed through Microsoft Excel 2013 software, obtaining the following results: Parent / Parent relationship (85\%), mental deficiency (45\%), waiting period is not reasonable $(75 \%)$, family members rarely $(60 \%)$, the staff is more or less $(65 \%)$ and the quality of service is more or less (95\%). It was observed that the time of service, waiting period, difficulty in obtaining information, inappropriate services, inadequate listening and reception are the main factors responsible for the difficulties of the reception offered by the health services to disabled users.
\end{abstract}

Keywords: Welcoming; Access to health services; User satisfaction.

\footnotetext{
${ }^{1}$ Faculdade Independente do Nordeste. E-mail: rubra2011@hotmail.com;

${ }^{2}$ Graduação em História pela Universidade Estadual do Sudoeste da Bahia . Pós - graduação em Psicopedagogia Clínica e Institucional pela Universidade Integrada Simonsen. Pós - Graduação em Educação Inclusiva pela Universidade Estadual da Bahia. Tem experiência na área de História, com ênfase em Interprete de Lingua de Sinais. Graduada em Letras Vernácula pela UESC no sistema UAB. É mestre em Linguística pela Universidade Estadual do Sudoeste da Bahia UESB. Bacharel em Letras Libras Universidade Estadual de Santa Catarina (UFSC).
} 
Id on Line Revista Multidisciplinar e de Psicologia

Id on Line Multidisciplinary and Psycology Journal

\section{Introdução}

De acordo com Reis e Silva (2012) as pessoas com necessidades especiais - PNE são aquelas que apresentam de modo permanente ou transitório, perdas ou reduções de estrutura, ou funções anatômicas, psicológicas, fisiológicas ou mentais, resultando na dificuldade para determinadas atividades, dentro do padrão considerado normal para o ser humano.

Segundo o decreto-lei $\mathrm{n}^{\mathrm{o}}$ 3.298, de 20 de dezembro de 1999 (BRASIL, 1999) a deficiência permanente é aquela que ocorre ou se estabilizou durante um período de tempo suficiente para não permitir recuperação ou ter probabilidade de que se altere, apesar de novos tratamentos. Deficiência Transitória consiste no acometimento de pessoas que estão debilitadas de suas funções físicas por um período determinado de tempo, ou seja, após o processo de reabilitação terão suas condições fisiológicas, psicológicas e anatômicas adequadas.

O Instituto Brasileiro de Geografia e Estatística (IBGE), revelou em 2010 que 45 milhões de pessoas, o que correspondente a $24 \%$ da população brasileira, se declaram portadoras de algum tipo de deficiência. Este resultado faz com que a necessidade de modificações na sociedade seja reforçada, principalmente na formação de profissionais voltados para esse contingente, para que tenham garantidos seus direitos de cidadania (AMARAL, 2012).

A promoção do desenvolvimento de pessoas com necessidades especiais é feita através do compromisso integral, baseado na garantia dos direitos do indivíduo à saúde em consonância aos princípios de universalidade, integridade e equidade; preconizados pelo SUS (CASTRO, 2011).

Indivíduos com deficiência constituem um grupo heterogêneo e as ações de saúde para esse segmento devem considerar as diferentes necessidades. A acessibilidade aos serviços de saúde é um direito de todos, sendo assim, as pessoas portadoras de necessidades especiais devem ser acolhidas e atendidas em tais serviços (MACÊDO et al., 2014).

Ainda existem grandes limitações no que se refere à garantia do acesso aos serviços de saúde pelas pessoas portadoras de necessidades especiais, demonstrando que a integralidade na atenção à saúde ainda não se efetiva. É necessária a ampliação de debates sobre acessibilidade das pessoas com deficiência para que ações transformadoras sejam planejadas e se tenha um serviço de saúde mais resolutivo (MACÊDO et al., 2014). 
Para Fonseca et al. (2013), o ambiente hospitalar é um exemplo de serviço de saúde com dificuldades para o acesso de pessoas com necessidades especiais. Estes locais normalmente não dispõem de cadeiras de rodas e macas de transporte destinadas aos pacientes com necessidades especiais e a estrutura física nem sempre é adequada. Além disso, existem lacunas na realização dos procedimentos básicos, a exemplo de um encaminhamento para o banho, pois os banheiros são estreitos e dificultam as manobras de certos equipamentos.

Paralelo a isto, estão os profissionais de saúde que nem sempre sentem-se preparados para prestarem atendimento ao paciente com necessidades especiais. Frente a isto, justifica-se a importância deste estudo, pois a investigação das dificuldades enfrentadas por pessoas com deficiência no acesso aos serviços de saúde pode fornecer subsídios para o planejamento em saúde, estruturando e melhorando os serviços de forma que atendam a esse grupo populacional de maneira adequada.

Acredita-se que os usuários com necessidades especiais enfrentam dificuldades no acolhimento dentro do serviço de saúde, pois os recintos que se destinam ao acolhimento não estão devidamente preparados e equipados para atender de forma integral e digna aos usuários com necessidades especiais. Nesta perspectiva, têm-se por questão problema, quais são as principais dificuldades encontradas pelos usuários com necessidades especiais no acolhimento de serviço de saúde?

Para responder a este questionamento o presente estudo tem por objetivo analisar as diversas formas de acolhimento prestadas aos usuários com necessidades especiais que utilizam os serviços de saúde da cidade de Vitória da Conquista, e, para isso, tem como objetivos específicos: descrever as ações envolvidas no acolhimento aos usuários com necessidades especiais; apontar as principais dificuldades do profissional de saúde ao acolher o pacientes com necessidades especiais e propor reflexões que sirvam para promover melhorias nos serviços de atendimento, aos usuários com necessidades especiais.

\section{Material e Método}

O estudo é caracterizado com exploratório de abordagem quantitativa. Segundo Marconi e Lakatos (2007), a pesquisa quantitativa tem como objetivo principal transformar opiniões e informações em números para possibilitar a classificação e análise. 
Id on Line Revista Multidisciplinar e de Psicologia

Id on Line Multidisciplinary and Psycology Journal

A pesquisa foi realizada numa escola pública de ensino fundamental no município de Vitória da Conquista, situado na região Sudoeste da Bahia. De acordo com o censo demográfico realizado pelo Instituto Brasileiro de Geografia e Estatística (IBGE, 2010), a população do município era de 306.866 habitantes.

A amostra para o estudo foi constituída de 20 pessoas de ambos os sexos, sendo essas familiares de uma pessoa deficiente cursando o ensino fundamental de uma escola pública do município de Vitória da Conquista.

Foram estabelecidos os seguintes critérios de inclusão: familiares de alunos com deficiência de uma escola pública de ensino fundamental do município de Vitória da ConquistaBA e que aceitarem participar espontaneamente desse estudo. Como critérios de exclusão estabeleceram-se: familiares de alunos que não cursam o ensino fundamental ou que não aceitarem participar da pesquisa.

Para a realização da coleta de dados foi utilizado um questionário objetivo, composto por 20 questões quantitativas objetivando conhecer a percepção dos familiares de alunos com necessidades especiais acerca do tratamento e dos serviços oferecidos aos seus parentes pelas instituições de saúde. A coleta foi efetuada em dias e horários estabelecidos pelo diretor da instituição. Os dados foram analisados através do software Microsoft Excel 2013.

Os participantes assinaram um termo de consentimento livre esclarecido (TCLE), que lhes garante o sigilo das informações, respeitando as determinações éticas de pesquisas com seres humanos, fundamentadas na Resolução 466/12 do Conselho Nacional de Saúde. A realização desta pesquisa ocorreu após aprovação do Comitê de Ética em Pesquisa da Faculdade Independente do Nordeste (FAINOR).

\section{Resultados}

Em relação à avaliação dos familiares de uma pessoa deficiente quanto ao seu parente e ao tratamento oferecido pelas unidades de saúde observou-se o predomínio dos seguintes dados: grau de parentesco Pai/Mãe (85\%), deficiência mental (45\%), com frequência razoável acompanham o seu parente (75\%), frequentemente têm contato com a equipe (50\%), estão 
satisfeitos com as medidas de privacidade (60\%), o tempo de atendimento é superior a 30 minutos $(95 \%)$ e o período de espera não é nada razoável (75\%), como mostra a Tabela 1.

Tabela 1 - Avaliação dos familiares de uma pessoa deficiente quanto ao seu parente e ao tratamento oferecido pelas unidades de saúde - BA, 2016.

\begin{tabular}{|c|c|c|}
\hline Variáveis & $\mathbf{n}$ & $\%$ \\
\hline \multicolumn{3}{|l|}{ Grau de parentesco } \\
\hline Pai/Mãe & 17 & 85,0 \\
\hline Irmão/Irmã & 1 & 5,0 \\
\hline Tio/Tia & 0 & 0,0 \\
\hline Outros & 2 & 10,0 \\
\hline \multicolumn{3}{|l|}{ Deficiência do seu parente } \\
\hline Deficiência física & 3 & 15,0 \\
\hline Deficiência auditiva & 4 & 20,0 \\
\hline Deficiência visual & 4 & 20,0 \\
\hline Deficiência mental & 9 & 45,0 \\
\hline Deficiência múltipla & 0 & 0,0 \\
\hline \multicolumn{3}{|c|}{ Frequência de acompanhamento } \\
\hline Muito frequentemente & 3 & 15,0 \\
\hline Com frequência razoável & 15 & 75,0 \\
\hline Raramente & 2 & 10,0 \\
\hline Dificilmente & 0 & 0,0 \\
\hline \multicolumn{3}{|c|}{ Frequência de contato com a equipe } \\
\hline Raramente & 3 & 15,0 \\
\hline Mais ou menos & 5 & 25,0 \\
\hline Frequentemente & 10 & 50,0 \\
\hline Muito frequentemente & 2 & 10,0 \\
\hline \multicolumn{3}{|c|}{ Satisfação com as medidas de privacidade } \\
\hline Insatisfeito & 3 & 15,0 \\
\hline Indiferente & 5 & 25,0 \\
\hline Satisfeito & 12 & 60,0 \\
\hline Muito satisfeito & 0 & 0,0 \\
\hline \multicolumn{3}{|l|}{ Tempo de atendimento } \\
\hline Fui atendido imediatamente & 0 & 0,0 \\
\hline Até 5 minutos & 0 & 0,0 \\
\hline Mais de 10 até 15 minutos & 1 & 5,0 \\
\hline Mais de 30 minutos & 19 & 95,0 \\
\hline \multicolumn{3}{|l|}{ Período de espera } \\
\hline Nada razoável & 15 & 75,0 \\
\hline Não muito razoável & 1 & 5,0 \\
\hline Razoável & 4 & 20,0 \\
\hline Muito razoável & 0 & 0,0 \\
\hline Total & 20 & 100,0 \\
\hline
\end{tabular}

Fonte: Dados coletados pelos autores

Quanto ao acolhimento oferecido pelas unidades de saúde verificou-se que: os familiares raramente se sentiam respeitados (60\%), mais ou menos e raramente tinham seus direitos violados (35\%), eram escutados mais ou menos pelo atendente $(95 \%)$, mais ou menos 
tinham dificuldades para obterem informações (50\%), mais ou menos e inapropriados são os serviços de saúde (50\%), mais ou menos os serviços são adequados (80\%) e o acolhimento da equipe é mais ou menos (65\%), de acordo com a Tabela 2.

Tabela 2 - Acolhimento oferecido pelas unidades de saúde - BA, 2016.

\begin{tabular}{|c|c|c|}
\hline Variáveis & $\mathbf{n}$ & $\%$ \\
\hline \multicolumn{3}{|l|}{ Tratamento oferecido pela equipe } \\
\hline Nunca me senti respeitado & 2 & 10,0 \\
\hline Raramente me senti respeitado & 12 & 60,0 \\
\hline Frequentemente me senti respeitado & 2 & 10,0 \\
\hline Sempre me senti respeitado & 4 & 20,0 \\
\hline \multicolumn{3}{|l|}{ Violação dos direitos } \\
\hline Muito frequentemente & 2 & 10,0 \\
\hline Frequentemente & 4 & 20,0 \\
\hline Mais ou menos & 7 & 35,0 \\
\hline Raramente & 7 & 35,0 \\
\hline \multicolumn{3}{|l|}{ Escuta oferecida pelo atendente } \\
\hline Não me escutou de forma alguma & 0 & 0,0 \\
\hline Mais ou menos & 19 & 95,0 \\
\hline Me escutou bastante & 1 & 5,0 \\
\hline Me escutou o tempo todo & 0 & 0,0 \\
\hline \multicolumn{3}{|l|}{ Dificuldade para obter informações } \\
\hline Sempre & 6 & 30,0 \\
\hline Frequentemente & 2 & 10,0 \\
\hline Mais ou menos & 10 & 50,0 \\
\hline Raramente & 2 & 10,0 \\
\hline \multicolumn{3}{|l|}{ Apropriação dos serviços de saúde } \\
\hline Muito inapropriados & 0 & 0,0 \\
\hline Inapropriados & 10 & 50,0 \\
\hline Mais ou menos & 10 & 50,0 \\
\hline Apropriados & 0 & 0,0 \\
\hline \multicolumn{3}{|l|}{ Serviço adequado } \\
\hline Não, de forma alguma & 2 & 10,0 \\
\hline Mais ou menos & 16 & 80,0 \\
\hline Em geral, sim & 2 & 10,0 \\
\hline Sim, com certeza & 0 & 0,0 \\
\hline \multicolumn{3}{|l|}{ Acolhimento da equipe } \\
\hline Nada amigável & 3 & 15,0 \\
\hline Não muito amigável & 2 & 10,0 \\
\hline Mais ou menos & 13 & 65,0 \\
\hline Amigável & 2 & 10,0 \\
\hline Total & 20 & 100,0 \\
\hline
\end{tabular}

Fonte: Dados coletados pelos autores

Acerca da caracterização do serviço prestado pelas unidades de saúde identificou-se que: a competência da equipe é mais ou menos (85\%), possuem mais ou menos os benefícios do atendimento (55\%), a qualidade do serviço é mais ou menos (95\%), são indiferentes quanto 
à satisfação com o serviço prestado (70\%), são indiferentes quanto à satisfação com o conforto do ambiente (50\%) e são indiferentes quanto à satisfação com o atendimento (80\%), conforme a Tabela 3

Tabela 3 - Caracterização do serviço prestado pelas unidades de saúde - BA, 2016.

\begin{tabular}{|c|c|c|}
\hline Variáveis & $\mathbf{n}$ & $\%$ \\
\hline \multicolumn{3}{|l|}{ Competência da equipe } \\
\hline Muito incompetente & 0 & 0,0 \\
\hline Incompetente & 1 & 5,0 \\
\hline Mais ou menos & 17 & 85,0 \\
\hline Competente & 2 & 10,0 \\
\hline \multicolumn{3}{|c|}{ Benefícios do atendimento } \\
\hline Não, de forma alguma & 1 & 5,0 \\
\hline Mais ou menos & 11 & 55,0 \\
\hline Sim, de alguma forma & 7 & 35,0 \\
\hline Sim, com certeza & 1 & 5,0 \\
\hline \multicolumn{3}{|l|}{ Qualidade do serviço } \\
\hline Ruim & 1 & 5,0 \\
\hline Mais ou menos & 19 & 95,0 \\
\hline Boa & 0 & 0,0 \\
\hline Excelente & 0 & 0,0 \\
\hline \multicolumn{3}{|c|}{ Satisfação com o serviço prestado } \\
\hline Muito insatisfeito & 0 & 0,0 \\
\hline Insatisfeito & 6 & 30,0 \\
\hline Indiferente & 14 & 70,0 \\
\hline Satisfeito & 0 & 0,0 \\
\hline \multicolumn{3}{|c|}{ Satisfação com o conforto do ambiente } \\
\hline Muito insatisfeito & 0 & 0,0 \\
\hline Insatisfeito & 2 & 10,0 \\
\hline Indiferente & 10 & 50,0 \\
\hline Satisfeito & 8 & 40,0 \\
\hline \multicolumn{3}{|c|}{ Satisfação com o atendimento } \\
\hline Muito insatisfeito & 0 & 0,0 \\
\hline Insatisfeito & 4 & 20,0 \\
\hline Indiferente & 16 & 80,0 \\
\hline Satisfeito & 0 & 0,0 \\
\hline Total & 20 & 100,0 \\
\hline
\end{tabular}

Fonte: Dados coletados pelos autores 
Id on Line Revista Multidisciplinar e de Psicologia

Id on Line Multidisciplinary and Psycology Journal

\section{Discussão}

Quanto ao grau de parentesco e ao tipo de deficiência apresentada pelo seu parente, houve predominância de pai/mãe e deficiência mental, respectivamente. No entanto, não há na literatura estudos que expliquem o acompanhamento das pessoas com necessidades especiais aos serviços de saúde pelos pais nem mesmo o destaque da deficiência mental entre as demais classificações das deficiências.

Ao que se refere à frequência de acompanhamento do seu ente e frequência de contato com a equipe de saúde, os familiares responderam frequência razoável e frequentemente, na devida ordem. Além disso, os entrevistados também afirmaram estarem satisfeitos com as medidas de privacidade oferecidas pelos serviços de saúde. Tal dado mostra-se satisfatório, visto que oferecer medidas de privacidade no momento do atendimento demonstra respeito pelo paciente.

A respeito do tempo de atendimento e o período de espera, os familiares de pessoas com deficiência responderam mais de 30 minutos e nada razoável, respectivamente.

O estudo realizado por Pessalacia et al. (2016) com familiares de pessoas com Síndrome de Down do município de Divinópolis-MG mostrou que o tempo de atendimento e o período de espera foram elencados como obstáculos para o uso dos serviços de saúde. Além disso, destacam a importância do período de espera para os pacientes com algum tipo de deficiência, pois podem apresentar necessidades especiais de descanso, alimentação ou higiene.

Quando questionados sobre se sentirem respeitados os participantes afirmaram raramente e, com relação a serem escutados e acolhidos pela equipe dos serviços de saúde bem como se encontram dificuldades para obterem as informações desejadas eles responderam mais ou menos.

Machado (2012) destaca que o acolhimento objetiva a escuta, a importância aos relatos do paciente ou da sua família e, o reconhecimento das suas necessidades, o respeito às diferenças, sempre priorizando o diálogo e a criação de vínculo entre os usuários e os trabalhadores da área de saúde.

$\mathrm{O}$ acolhimento especializado, a escuta qualificada e o respeito são fatores que interferem de forma positiva na qualidade de vida das pessoas com necessidades especiais. Além disso, 
Id on Line Revista Multidisciplinar e de Psicologia

Id on Line Multidisciplinary and Psycology Journal

promovem o suprimento de suas necessidades principais e proporcionam a adaptação e o tratamento adequados (MARTINS; CORTÊS; VALENTIM, 2014).

O estudo realizado por Tedesco e Junges (2013) em unidades básicas de saúde de Porto Alegre mostrou que o acolhimento é uma diretriz operacional capaz de modificar o modelo técnico-assistencial do SUS, garantindo o acesso universal e a qualificação das relações.

Em um estudo realizado no Rio Grande do Norte por Resende, Nóbrega e Moreira (2014) foi evidenciado que os usuários dos serviços de saúde não se sentiam acolhidos e nem tinham sua condição reconhecida e valorizada pelos profissionais. Dessa forma, afirmam que o processo de humanização deve ser consolidado de maneira inclusiva, na qual os profissionais de saúde possam vivenciar práticas específicas referentes à cultura de inclusão.

Quanto à apropriação dos serviços prestados pelas unidades de saúde bem como a adequação destes serviços, os entrevistados afirmaram ser inapropriados e mais ou menos, nesta ordem.

Em seu estudo, Resende, Nóbrega e Moreira (2014) afirmam que a falta de informação dos profissionais sobre as especificidades do paciente portador de necessidades especiais e o comprometimento da relação profissional-paciente são fatores que indicam a precariedade e a inadequação dos serviços de saúde prestados para pessoas com deficiência.

A presença de estigmas, violência institucional e relações marcadas pelo modelo biomédico são fatores que interferem de maneira negativa no cuidado efetivo à saúde de pessoas portadoras de necessidades especiais. Apontam a construção de relações muito próximas e o estabelecimento de vínculos entre profissional e paciente como medidas que permitem a identificação de riscos e o estabelecimento de intervenções (VIEIRA; FAVORETO, 2016).

Quando indagados a respeito da violação dos direitos os participantes afirmaram raramente. O estudo realizado por Nicolau, Schraiber e Ayres (2013) com portadoras de deficiência que frequentam uma unidade de saúde em São Paulo apontou que apesar de as políticas de saúde apresentarem em pauta os direitos das pessoas portadoras de deficiência pouco se sabe a respeito de como tais direitos são articulados nos procedimentos assistenciais e nas ações de promoção à saúde e prevenção de doenças dos serviços de saúde.

Com relação à avaliação da competência da equipe dos serviços de saúde, os familiares de pessoas portadoras de necessidades especiais responderam mais ou menos. 
Id on Line Revista Multidisciplinar e de Psicologia

Id on Line Multidisciplinary and Psycology Journal

Em um estudo realizado por Martins, Cortês e Valentim (2014) no estado de Mato Grosso evidenciou-se que o planejamento em saúde voltado para ações específicas aos indivíduos com necessidades especiais corrobora para o conhecimento dos seus direitos bem como para o comprometimento com o cuidado prestado, possibilitando-os uma vida mais feliz e digna.

O despreparo dos profissionais de saúde em prestar serviços aos pacientes com necessidades especiais associado à não utilização da escuta qualificada e do acolhimento como formas de estabelecerem uma comunicação eficaz, apontam para a necessidade de inserção desta temática na educação permanente, objetivando capacitar os profissionais e reorientar os serviços de saúde (TEDESCO; JUNGES, 2013).

Ao que se refere aos benefícios do atendimento e à qualidade do serviço oferecido pelas unidades de saúde os entrevistados responderam mais ou menos.

A qualidade dos serviços e o consequente benefício do atendimento em saúde são frutos de um instrumento importante: a comunicação. Quando realizada de forma efetiva colabora para a humanização das interações e auxilia no suprimento das necessidades do cliente (GIUSTINA; CARNEIRO; SOUZA, 2015).

Em seu estudo, Giustina, Carneiro e Souza (2015) afirmam que para que ocorra a melhoria da qualidade dos serviços e do atendimento em saúde destinados aos pacientes com necessidades especiais é necessária a capacitação dos profissionais e a valorização desta qualificação na admissão de novos funcionários, para que os mesmos possam prestar aos pacientes com deficiência uma assistência eficaz, baseada nos princípios do SUS.

A respeito da satisfação com o serviço prestado, o conforto do ambiente e o atendimento oferecido os participantes se mostraram indiferentes.

A satisfação de usuários dos serviços de saúde depende da associação de alguns fatores como: características do atendimento; adesão ao tratamento proposto; competência da equipe; acessibilidade ao serviço; informação recebida e qualidade da relação profissional-paciente (SILVA et al., 2012).

O estudo realizado por Silva et al. (2016) em uma unidade básica de saúde do Estado de Goiás afirma que o sistema de saúde apresenta dificuldades que impedem a satisfação da população quanto a assistência prestada. 
Id on Line Revista Multidisciplinar e de Psicologia

Id on Line Multidisciplinary and Psycology Journal

\section{Considerações Finais}

O presente estudo nos permitiu elencar os seguintes itens como os principais fatores responsáveis pelas dificuldades do acolhimento de pessoas com necessidades especiais nos serviços de saúde: tempo de atendimento; período de espera; dificuldade para obter informações; serviços inapropriados e escuta e acolhimento inadequados.

Diante do exposto, é de grande relevância que novas políticas públicas sejam inseridas para melhorar a qualidade do acolhimento oferecido pelos serviços de saúde às pessoas com deficiência, bem como programas específicos de educação permanente para os profissionais da equipe de saúde, objetivando prepará-los para lidar com as necessidades especiais desses pacientes e prestar uma assistência embasada nos princípios preconizados pelo SUS.

Em suma, esta pesquisa fornece informações referentes ao acolhimento oferecido pelos serviços de saúde aos indivíduos com necessidades especiais. Estes dados poderão ser utilizados futuramente para o embasamento de novos estudos e possíveis ações para tornar os serviços de saúde acolhedores e resolutivos para as pessoas com deficiência.

\section{Referências}

AMARAL, Fabienne Louise Juvêncio dos Santos et al. Fatores associados com a dificuldade no acesso de idosos com deficiência aos serviços de saúde. Ciênc. saúde coletiva, v. 17, n. 11, p. 2991-3001, 2012.

BRASIL. Decreto-Lei $\mathbf{n}^{\mathbf{0}} 3.298$ de 20 de dezembro de 1999. Brasília, DF, 1999. Disponível em: http://www.planalto.gov.br/ccivil_03/decreto/d3298.htm. Acesso em: 25 de maio de 2016.

CASTRO, Shamyr Sulyvan et al. Acessibilidade aos serviços de saúde por pessoas com deficiência. Revista de Saúde Pública, v. 45, n. 1, p. 99-105, 2011.

DE ANDRADE MARCONI, Marina; LAKATOS, Eva Maria. Metodologia do trabalho científico: procedimentos básicos, pesquisa bibliográfica, projeto e relatório, publicações e trabalhos científicos. Atlas, 2007.

DE MACÊDO, Gilsene Caroline Ponte et al. Dificuldades de Acessibilidades nos Serviços De Saúde Vividas por Pessoas com Deficiência: Uma Revisão Integrativa. In: $11^{\circ}$ Congresso Internacional da Rede Unida. 2014. 
Id on Line Revista Multidisciplinar e de Psicologia

Id on Line Multidisciplinary and Psycology Journal

DELlA GIUSTINA, Flávia Pinheiro; DAS NEVES CARNEIRO, Denise Medeiros; DE SOUZA, Ruana Medeiros. A Enfermagem e a DeficiêNcia Auditiva: assistencia ao surdo. Revista de Saúde da Faciplac, v. 2, n. 1, 2015.

FONSECA, Luciana Mascarenhas et al. Implicações Sobre Deficiência Intelectual e Longevidade: Projeto piloto de Intervenção em Casos de Senilidade. Apae Ciência, v. 1, n. 2, 2013.

IBGE - Instituto Brasileiro de Geografia e estatística. População idosa no Brasil no Censo Demográfico de 2010. Disponível em: http://www.ibge.gov.br. Acesso em: 20 de maio de 2016.

MACHADO, Suelen. Desafios e possibilidades da triagem na emergência. 2012.

MARTINS, Rejane; CORTÊS, Mayra A.; VALENTIM, Flavio César Vieira. Educação em saúde e o cuidar pelo enfermeiro na área da saúde mental: enfoque em indivíduos com déficit cognitivo. Revista Eletrônica Interdisciplinar, v. 1, n. 11, 2014.

NICOLAU, Stella Maris; SCHRAIBER, Lilia Blima; AYRES, José Ricardo de Carvalho Mesquita. Mulheres com deficiência e sua dupla vulnerabilidade: contribuições para a construção da integralidade em saúde. Ciência \& Saúde Coletiva, v. 18, n. 3, p. 863-872, 2013.

PESSALACIA, Juliana Dias Reis et al. Experiências de acesso a serviços primários de saúde por pessoas com Síndrome de Down. Revista de Enfermagem do Centro-Oeste Mineiro, v. 5, n. 3, 2016.

REIS, Drielly Santos; DA SILVA, Dayana Matias Coelho. Recrutamento e Seleção de Portadores de Necessidades Especiais-PNE. SIMPÓSIO DE EXCELÊNCIA EM GESTÃO E TECNOLOGIA, v. 8, p. 1-16, 2012.

RESENDE, Adara Cabral; DA NÓBREGA, Simone; MOREIRA, Tomaz. Experiência das Pessoas com Deficiência nos Serviços de Saúde: Contribuição para a Formação Profissional. Blucher Medical Proceedings, v. 1, n. 2, p. 61-61, 2014.

SILVA, Daniele Cristina Nascimento et al. ACESSIBILIDADE DE PORTADORES DE DEFICIÊNCIA FÍSICA OU MOBILIDADE REDUZIDA NA UNIDADE BÁSICA DE SAÚDE JONAS MANOEL DIAS EM SÃO LUÍS DE MONTES BELOS-GO. Revista Eletrônica Faculdade Montes Belos, v. 8, n. 3, 2016.

TEDESCO, Janaina dos Reis; JUNGES, José Roque. Desafios da prática do acolhimento de surdos na atenção primária. 2013.

VIEIRA, Daniela Koeller Rodrigues; FAVORETO, César Augusto Orazen. Narrativas em saúde: refletindo sobre o cuidado à pessoa com deficiência e doença genética no Sistema Único de Saúde (SUS). Interface-Comunicação, Saúde, Educação, v. 20, n. 56, p. 89-98, 2016.

\section{Como citar este artigo (Formato ABNT):}

GOTADO, R.G.; ALMEIDA, M.A.A.P.T. O Acolhimento oferecido pelos Serviços de Saúde às pessoas com Necessidades Especiais . Id on Line Revista Multidisciplinar e de Psicologia, Nov-Dez. de 2016, vol.10, n.32, p. 126-137. ISSN: 1981-1179.

Recebido: 09/11/2016

Aceito: 16/11/2016 